\title{
GEODE OR SUBCHONDRAL BONE CYST.
}

\section{Orthopaedics}

Dr. Devi Saranya . CRRI - Intern, Chettinad Hospital And Research Institute, Chettinad Academy Of B

Research And Education, Kelambakkam, Chengalpattu District, Pin - 603103.

Dr.

Venkatachalam.

$\mathbf{K}^{*}$

Prof. And HOD, Department Of Orthopaedics, Medical Superintendent, Chettinad Hospital And Research Institute, Chettinad Academy Of Research And Education, Kelambakkam, Chengalpattu District, Pin - 603103.*Corresponding Author

Dr. Ampalaya

Manu R

MS (Ortho) PG, Department Of Orthopaedics, Chettinad Hospital And Research Institute, Chettinad Academy Of Research And Education, Kelambakkam, Chengalpattu District, Pin - 603103 .

Dr. Kamala

Roshini M

CRRI - Intern, Chettinad Hospital And Research Institute, Chettinad Academy Of Research And Education, Kelambakkam, Chengalpattu District, Pin - 603103.

\section{ABSTRACT}

Geode or subchondral bone cyst is a lytic lesion in the peri-articular area and this condition is seen as a cyst or cyst erosion in a bone end. Basically, a cyst is lined by epithelium but subchondral bone cyst is not lined by epithelium. Subchondral bone cysts are common in various articular diseases.

\section{KEYWORDS}

Geode, Subchondral Cyst, Osteoarthritis Knee.

\section{INTRODUCTION:}

Geode or subchondral bone cyst is a lytic lesion in the peri-articular area and this condition is seen as a cyst or cyst erosion in a bone end. It was initially discovered in the 1940s. Generally, the term Geode is borrowed from geology, meaning a rounded pocket of gas in a mineral specimen. ${ }^{[1]}$

\section{Composition:}

Basically a cyst is lined by epithelium but subchondral bone cyst is not lined by epithelium. In the weight bearing joints, such as knees, hip, shoulders etc. the subchondral bone mimics the action of an effective shock absorber. The fluid inside subchondral bone cyst is hyaluronic acid, a component found in synovial fluid, which is the thick substance that lubricates joints, allowing the bones to slide one over other with minimal friction.

\section{Pathogenesis:}

Geode formation takes place possibly when synovial fluid is forced into the subchondral bone causing a cystic collection of joint fluid or following a contusion, in which the contused bone forms. ${ }^{[1}$ Subchondral bone cyst may result from pressure or stress on articular cartilage and subchondral bone which leads to synovial fluid intrusion. Subchondral radiolucencies are found uniformlywithout cavitation and epithelial lining. Bone cysts frequently occurs in association with joint space loss and bone eburnation, communication with the joint cavity may or may not be present. ${ }^{[1]}$

\section{Risk factors:}

Sex(women), hereditary, joint alignment and space, obesity, smoking, activity and injury. ${ }^{[2]}$

\section{Associated illness:}

Subchondral bone cyst are common in many type of articular disease particularly in osteoarthritis, rheumatoid arthritis and osteonecrosis. ${ }^{[3]}$ Subchondral bone cyst are mainly seen in degenerative joint disease, avascular necrosis, calcium pyrophosphate dihydrate crystal deposition disease.

\section{Symptoms:}

Rarely, the symptoms are discomfort, pain, lack of flexibility in the joint, swelling or bulging around the joint, subchondral bone cysts are associated with osteoarthritis.

Investigation:

3D imaging techniques such as CT, MRI if patient is a known case of osteoarthritis and pre-clinical imaging to monitor the initiation and progression of subchondral bone cyst occuring within an established rodent model of knee osteoarthritis.

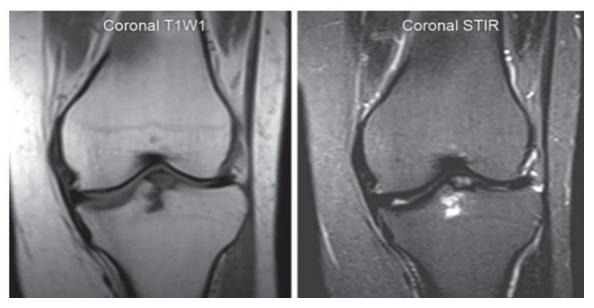

Fig 1(a) and 1(b) Sub-articular T1WI shows hypo intense lesion measuring $6 \mathrm{~mm}$ just beneath the tibial spine appearing hyper intense on STIR image.
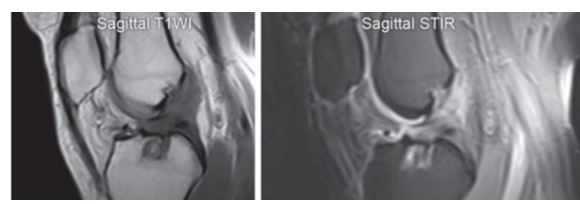

Fig 2(a) and 2(b ) : Sub-articular geode appearing hypo intense on sagittal T1W image and hyper intense on STIR images.

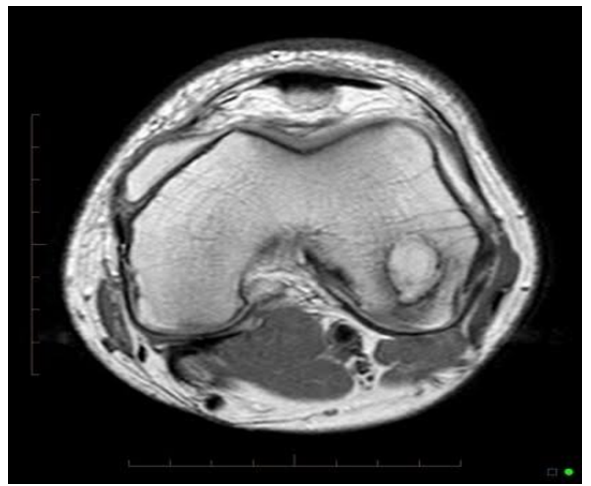

Fig 3 : Cartilage defect with subadjacent subchondral cyst involving the lateral femoral condyle.

Differential diagnosis include epiphyseal lesion. 


\section{Treatment}

- It starts with life style modification including weight loss in patient with osteoarthritis.

- High impact activities such as swimming or cycling should be prevented.

- Non operative management includes NSAIDS such as ibuprofen which may temporarily reduce the symptoms, ultrasonic therapy may helpful. ${ }^{[4]}$

- Operative management includes drilling and bone grafting.

\section{DISCUSSION :}

Geodes or subchondral cysts are radiolucent lesions adjacent to joints seen commonly in osteoarthritis, particularly in the hip, but are also noted in other articular conditions such as rheumatoid arthritis, osteonecrosis and calcium pyrophosphate disease. ${ }^{[5]}$ In rheumatoid arthritis, geodes are generally small; however, large geodes have been reported in other areas including the tibia, ulna and humerus and may reach sizes up to $13 \mathrm{~cm}$ in diameter. ${ }^{[6]}$ The pathogenesis in osteoarthritis is known to involve both bone contusion and extrusion of synovial fluid into the bone because of elevated intra-articular pressure; in rheumatoid arthritis, invasion of pannus may play a role. ${ }^{[7]}$

\section{CONCLUSION :}

Treatment for geodes is controversial; however, pathological fractures have been reported, prompting some to suggest that preventive surgical treatment should be considered for giant geodes. ${ }^{\left[{ }^{[}\right]}$

\section{REFERENCE:}

1. Stoufflet PE, Ohno-Machado L, Deibel SR, Lee D, Greenes RA. GEODE-CM: a statetransition framework for clinical management. InProceedings of the AMIA Annual Fall Symposium 1996 (p. 924). American Medical Informatics Association.

2. Amin, S., Niu, J., Guermazi, A., Grigoryan, M., Hunter, D. J., Clancy, M., Felson, D. T (2007). Cigarette smoking and the risk for cartilage loss and knee pain in men with knee osteoarthritis [Abstract]. Annals of the Rheumatic Diseases, 66(1), 18-22

3. Audrey, H. X., Bin Abd Razak, H. R., \& Andrew, T. H. C. (2014, January 24). The truth behind subchondral cysts in osteoarthritis of the knee. The Open Orthopaedics Journal, 8,7-10.

4. Resnick D. Degenerative disease of extraspinal locations. In: Resnick D, ed. Diagnosis of Bone and Joint Disorders. Philadelphia, PA: WB Saunders Company; 2002:1280-1286

5. Maher MM, Kennedy J, Hynes D, et al. Giant distal humeral geode. Skeletal Radiol. 2000;29:165-167.

6. Cohen AP, McWilliams TC. Giant geode (pseudocyst) formation of the femoral neck in a case of osteoarthritis. Rheumatology. 2000;39:443-444

7. Lohse A, Carbillet J-P, Onimus M, et al. Giant intraosseous cyst-like lesions in rheumatoid arthritis report of a case. Joint Bone Spine. 2003;70:67-70.

8. Nakagawa N, Abe S, Saegusa Y, et al. Giant geode at the olecranon in the rheumatoid elbow - two case reports. Clin Rheumatol. 2004;23:358-361. 